\title{
Erratum: Characteristics of chaos evolution in one-dimensional disordered nonlinear lattices [Phys. Rev. E 98, 052229 (2018)]
}

B. Senyange, B. Many Manda, and Ch. Skokos

(Received 24 May 2019; published 24 June 2019)

DOI: 10.1103/PhysRevE.99.069903

We have identified, in the original paper, confusing use of the symbol $\xi_{l}$ denoting the normalized energy [disordered KleinGordon (DKG) system] and norm density [disordered discrete nonlinear Schrödinger equation (DDNLS)], which only affects the presentation of some of the provided information about the setup of our numerical simulations.

Thus, the following changes should be made in the first paragraph of Sec. III: (a) $p_{l}= \pm \sqrt{2 \xi_{l}}$ should become $p_{l}= \pm \sqrt{2 \xi_{l} H_{K}}$ (line 4), (b) $\xi_{l}=1$ should become $\xi_{l} S=1$ (line 7), and (c) $H_{K}=L \xi_{l}$ should become $H_{K}$ (line 11).

Furthermore, in the last entry of the presentation of the studied cases in Sec. III A, $\xi_{l}$ should become $\xi_{l} H_{K}$ for the DKG system and $\xi_{l} S$ for the DDNLS model (e.g., Case $W 1_{K}: W=3, L=37, \xi_{l} H_{K}=0.01$; Case $S 1_{D}: W=3, \beta=0.5, L=21, \xi_{l} S=1$ ). We note that the rest of the paper is not affected by these corrections.

We thank J.-J. du Plessis for pointing out this issue to us. 